\title{
Diffusion Tensor Magnetic Resonance Imaging Strategies for Color Mapping of Human Brain Anatomy
}

\begin{abstract}
Background: A color mapping of fiber tract orientation using diffusion tensor imaging (DTI) can be prominent in clinical practice. The goal of this paper is to perform a comparative study of visualized diffusion anisotropy in the human brain anatomical entities using three different color-mapping techniques based on diffusion-weighted imaging (DWI) and DTI. Methods: The first technique is based on calculating a color map from DWIs measured in three perpendicular directions. The second technique is based on eigenvalues derived from the diffusion tensor. The last technique is based on three eigenvectors corresponding to sorted eigenvalues derived from the diffusion tensor. All magnetic resonance imaging measurements were achieved using a 1.5 Tesla Siemens Vision whole body imaging system. A single-shot DW echoplanar imaging sequence used a Stejskal-Tanner approach. Trapezoidal diffusion gradients are used. The slice orientation was transverse. The basic measurement yielded a set of 13 images. Each series consists of a single image without diffusion weighting, besides two DWIs for each of the next six noncollinear magnetic field gradient directions. Results: The three types of color maps were calculated consequently using the DWI obtained and the DTI. Indeed, we established an excellent similarity between the image data in the color maps and the fiber directions of known anatomical structures (e.g., corpus callosum and gray matter). Conclusions: In the meantime, rotationally invariant quantities such as the eigenvectors of the diffusion tensor reflected better, the real orientation found in the studied tissue.
\end{abstract}

Keywords: Color mapping, diffusion tensor, human brain anatomy, magnetic resonance imaging

\section{Introduction}

Diffusion-weighted (DW) magnetic resonance imaging (MRI) is recognized to have high sensitivity to water diffusion within living tissues; it is also strongly influenced by the structure and geometry features of the tissue related to measured diffusion behavior. DW-MRI has been demonstrated to be a valuable tool to study the human brain..$^{[1-13]}$ In the meantime, it has had successful achievements that were shown in the clinical application of DW imaging (DWI). For instance, it was demonstrated that the earliest stages of ischemic events and changes in the apparent diffusion coefficients (ADC) were recorded and corresponded to changes in the ratio of extracellular/intracellular water content; this was proved to be very supportive in the early detection and assessment of strokes. ${ }^{[14-19]}$ The brain white matter demonstrated that this application is more complicated by the diffusion behavior since it was found that diffusion

This is an open access journal, and articles are distributed under the terms of the Creative Commons Attribution-NonCommercialShareAlike 4.0 License, which allows others to remix, tweak, and build upon the work non-commercially, as long as appropriate credit is given and the new creations are licensed under the identical terms.

For reprints contact: reprints@medknow.com is much stronger along the white matter fiber tracts compared to perpendicular directions. ${ }^{[19]}$ Indeed this refers to diffusion anisotropy originated by tissue structures organization. Early quantitative and qualitative assessments of brain of neonatal and infant have showed that such diffusion anisotropy could be a sensitive marker of brain maturation. ${ }^{[2,8,9,12,13]}$

This successful clinical overview has impacted in such a way that a greater demand for studying the human brain connectivity which was introduced within a large range of investigation in the neuroscience field, the neurosciences community is involved in fields of research concerned with the functional and anatomical organization of the brain, studying focal lesion deficit, and brain development, ${ }^{[2,12,13]}$ especially magnetic resonance imaging (MRI) is a unique noninvasive investigation and assessment tool allowing to provide in vivo images of the brain for further analysis of brain structure and organization.

How to cite this article: Boujraf S. Diffusion tensor magnetic resonance imaging strategies for color mapping of human brain anatomy. J Med Sign Sens 2018;8:73-80.

\section{Saïd Boujraf ${ }^{1,2,3}$}

${ }^{\prime}$ Department of Biophysics and Clinical MRI Methods, Faculty of Medicine and Pharmacy, University of $\mathrm{Fez},{ }^{2}$ The Clinical Neuroscience Laboratory, Faculty of Medicine and Pharmacy, University of $\mathrm{Fez}$, ${ }^{3}$ Department Radiology and Clinical Imaging, University Hospital of Fez, Fez, Morocco
Address for correspondence: Dr. Saïd Boujraf, Department of Biophysics and Clinical MRI Methods, Faculty of Medicine and Pharmacy, University of Fez, BP. 1893; Km 2.200, Sidi Hrazem Road, Fez 30000, Morocco. E-mail:sboujraf@gmail.com

Website: www.jmss.mui.ac.ir DOI: 10.4103/jmss.JMSS_14_17 
Volumes of T1-weighted MRI scans have been used to achieve segmentation of brain structures allowing an automatic parcellation of diffusion tensor imaging (DTI) of the brain white matter and cortex. ${ }^{[2,12,13]}$ Such high-resolution MRI-based methods are highly suitable to investigate relationships between the anatomical regions within brain space.

In this context, DTI was developed, since it allows characterizing the diffusion behavior of water within tissue on a pixel by pixel basis. This goal is achieved by deriving diffusion coefficient describing the diffusion behavior in any direction in space from a matrix quantity that is the diffusion tensor. ${ }^{[2-13]}$ Indeed DTI provides unique quantitative information about brain tissue structure; with completely noninvasive mode while covering the entire brain. ${ }^{[1-19]}$ Consequently, diagonalizing the diffusion tensor allows calculating its eigenvalues and eigenvectors as well as sorted eigenvalues and eigenvectors. In such condition, the eigenvector corresponding to the largest eigenvalue is considered to represent the major diffusion direction within the concerned voxel. Considered that, diffusion anisotropy in white matter is originated by a larger limitation or restriction to diffusion across the fiber tissue axes compared to a long them. Hence, the primary eigenvector is to be considered pointing along the direction of a recognized fiber array navigating through the voxel. Thus, mapping directions are made up of the principal eigenvectors which can be generated, providing a striking visualization of the white matter tracts and corresponding orientation. ${ }^{[1-14]}$

The purpose of this work was to perform a comparative study of the diffusion anisotropy visualization of the human brain anatomical entities. This study consists of using a priori knowledge of geometry of white matter tissue while attempting to overcome the constraints related to the evaluation and comparison of three different color maps produced based on DWI and DTI. Those included the following:

1. The first technique is based on calculating a color map from diffusion-weighted images measured in three perpendicular directions of the measuring frame of reference $(\mathrm{x}, \mathrm{y}$, and $\mathrm{z})$

2. The second technique is based on the three derived eigenvalues from diffusion tensor, which we assign single color code to each eigenvalue obtained in a single pixel

3. The last technique is based on the three derived eigenvectors corresponding respectively to the sorted eigenvalues, that we assign a single color code to each eigenvector (obtained in a single pixel). The calculated qualitative maps obtained through each approach are compared to the reference anatomy slices abstained through brain dissection.

\section{Materials and Methods}

Background of the diffusion tensor theory

In DTI, the pixel intensity in the diffusion-weighted image and the diffusion tensor components $D_{i j}$ values is given by the relationship described in the diffusion tensor theory. ${ }^{[2,12,13,20,21]}$

The diffusion phenomenon is a physical process involving the water molecules' random motion in a given medium while being driven by kinetic energy theory. The statistical physics described the phenomenon by the first law of Fick. $^{[2,12,20-28]}$

$\mathrm{J}=-\mathrm{D} \nabla \mathrm{c}$

This law supports that a difference in concentration of a given moving molecules is creating a net flux from regions with a high concentration into the direction of regions with low concentration. Where $\mathrm{J}$ is the full flux, $\nabla \mathrm{c}$ is the concentration gradient, and finally, D is the diffusion coefficient. This, the diffusion coefficient $\mathrm{D}$ dependents on factors including dimensions of the diffusing molecule and the microstructure characteristics constituting the diffusion medium and its temperature. The diffusion coefficient is given by the Einstein equation:

$$
D=\frac{k_{\mathrm{B}} T}{6 \pi \eta r}
$$

Where $\mathrm{k}_{\mathrm{B}}$ is the Boltzmann constant, $\mathrm{T}$ and $\eta$ are the temperature measured in Kelvin and viscosity of the diffusion medium, respectively, $r$ is the radius of the diffusing molecule.

The net flux vanishes when concentration gradient becomes null; however, molecules do continue moving to support the thermodynamic equilibrium.

This phenomenon is known as Brownian motion and was not been described as a stochastic motion mode. Molecules movement could be represented by a Gaussian distribution displacement model depending on $\mathrm{D}$.

The probability for a given molecule to travel a distance $\mathrm{x}$ in the time $\mathrm{t}$ is defined by:

$P(x, t)=\frac{\exp \left(-x^{2} / 4 \mathrm{Dt}\right)}{(4 \pi \mathrm{Dt})^{1 / 2}}$

Indeed, the mean square displacement during the time $\Delta \mathrm{t}$ is given by:

$\left\langle x^{2}\right\rangle=2 \mathrm{D} \Delta \mathrm{t}$

The $\mathrm{x}$ distance was traveled by the diffusing molecule during $\Delta \mathrm{t}$. When there are not any boundaries for molecules motion, diffusion is described as free and follows the random Brownian motion model. This diffusion model is called isotropic.

For an isotropic medium, the diffusion tensor becomes: 
$\mathrm{D}=\mathrm{D}_{\mathrm{o}} . \mathrm{I}$

Where $\mathrm{D}_{\mathrm{o}}$ is the scalar diffusion coefficient and $\mathrm{I}$ is the unity matrix given by:

$I=\left(\begin{array}{lll}1 & 0 & 0 \\ 0 & 1 & 0 \\ 0 & 0 & 1\end{array}\right)$

A normalized DT, D/D $=\mathrm{D}_{\text {norm }}$ should reflect the specific constraint for an isotropic medium, namely that the three diagonal elements must be equal to each other $\left(\mathrm{D} / \mathrm{D}_{\mathrm{o}}=1\right)$ and the off-diagonal elements should cancel to zero. Significant deviations from this property are to be ascribed to systematic differences in sensitivity of the various MRI sequences to diffusion phenomena. Indeed, connecting the observed anisotropy for isotropic media to errors in calibration, misalignment, and cross-talk between the imaging and diffusion gradients has to be assessed. ${ }^{[2,12,20-28]}$

Nevertheless, when molecules movement is hindered by obstacles, the diffusion will demonstrate preferential direction; that is not associating barrier and the diffusion is said to be anisotropic. This last case is particularly important when studying the diffusion of water within human biological tissue since composed by organized structures that are guiding the water movement which is not diffusing in free mode. Often these structures are not visible with conventional MRI techniques since their dimension magnitudes are smaller than the resolution of a magnetic resonance image.

These aspects are important when interested by brain tissue magnetic resonance images. Thus, various brain tissues are highly characterized by their diffusion properties including white matter, gray matter, and cerebrospinal fluid. ${ }^{[2,12,20-28]}$

\section{Expression for the $\mathbf{b}$ matrix and diffusion tensor}

It is possible to compute the DT components from the signal intensity in the DW magnetic resonance images, thus, ${ }^{[20,21,29]}$

$$
\begin{aligned}
& \ln \left(\frac{\mathrm{I}(\mathrm{b})}{\mathrm{I}(0)}\right)= \\
& \quad-\gamma^{2} \cdot \int_{0}^{\mathrm{TE}}[\mathrm{F}(t)-2 \cdot \xi(t) \cdot \mathrm{f}] \mathrm{D}[\mathrm{F}(t)-2 \cdot \xi(t) \cdot \mathrm{f}]^{\mathrm{T}} \mathrm{d} t
\end{aligned}
$$

The $\mathrm{b}$ matrix can be calculated as follows:

$$
\mathrm{b}=b \gamma^{2} \cdot \int_{0}^{\mathrm{TE}}[\mathrm{F}(t)-2 \cdot \xi(t) \cdot \mathrm{f}]^{\mathrm{T}}[\mathrm{F}(t)-2 \cdot \xi(t) \cdot \mathrm{f}] \mathrm{d} t
$$

where

$$
\mathrm{F}(\mathrm{t})=\int_{0}^{t} \mathrm{G}\left(t^{\prime}\right) \mathrm{d} t^{\prime}, \quad \mathrm{f}=\mathrm{F}\left(\frac{1}{2} \mathrm{TE}\right)
$$

and $g$ is the gyromagnetic ratio, $G(t)=[G x(t), G y(t), G z(t)]$ the column vector representing the gradient pulses in the diffusion imaging sequence, TE the echo time, and $\xi(\mathrm{t})$ the Heaviside function with:

$\xi(t)=0$ when $t<\frac{1}{2} \mathrm{TE}$ and $\xi(t)=1$ when $t \geq \frac{1}{2} \mathrm{TE}$
The symmetric $3 \times 3$ tensor $\mathrm{D}$ is expressed in the laboratory frame $\mathrm{x}, \mathrm{y}, \mathrm{z}$ as follows:

$$
\mathrm{D}=\left[\begin{array}{lll}
\mathrm{D}_{\mathrm{xx}} & \mathrm{D}_{\mathrm{xy}} & \mathrm{D}_{\mathrm{xz}} \\
\mathrm{D}_{\mathrm{xy}} & \mathrm{D}_{\mathrm{yy}} & \mathrm{D}_{\mathrm{yz}} \\
\mathrm{D}_{\mathrm{xz}} & \mathrm{D}_{\mathrm{yz}} & \mathrm{D}_{\mathrm{zz}}
\end{array}\right]
$$

In the same frame of reference, expression ${ }^{[5]}$ can be rewritten under the form: ${ }^{[21,30]}$

$$
\begin{aligned}
& \ln \left(\frac{I(b)}{I(0)}\right)=\left\{-\sum_{i=1}^{3} \sum_{j=1}^{3} b_{i j} \cdot D_{i j}\right\} \\
& =-\left(\begin{array}{c}
b_{x x} \cdot D_{x x}+b_{y y} \cdot D_{y y}+b_{z z} \cdot D_{z z}+2 \cdot b_{x y} \cdot D_{x y}+ \\
2 \cdot b_{x z} \cdot D_{x z}+2 \cdot b_{y z} \cdot D_{y z}
\end{array}\right)
\end{aligned}
$$

$I$ (b) is the signal intensity for particular diffusion gradient sensitization, and $I(0)$ is the signal intensity measured in the absence of the diffusion gradient sensitization.

The elements of the $b$ matrix $b_{1}$ are computed from Eqs. 6 and 7, with a $G(t)$ that accurately reflects all gradients switched on at any time during the run of the DT MRI sequence.

\section{Eigenvalues}

In the previous section DT, D was expressed in the frame of reference of measuring laboratory. After diagonalizing the DT and sorting the calculated eigenvalues, we obtained the diagonal tensor diffusivity, $\lambda$, expressed in the following:

$$
\lambda=\left[\begin{array}{ccc}
\lambda_{1} & 0 & 0 \\
0 & \lambda_{2} & 0 \\
0 & 0 & \lambda_{3}
\end{array}\right]
$$

For an isotropic medium, Eq. 10 becomes $1=\lambda_{\mathrm{o}}$. I, where I is the unit tensor and

$\lambda_{0}=\frac{1}{3} \sum_{\mathrm{k}=1}^{3} \lambda_{\mathrm{k}}=\frac{1}{3} \sum_{\mathrm{k}=1}^{3} \mathrm{D}_{\mathrm{kk}}=\frac{1}{3} \operatorname{Tr}(\mathrm{D})$

Where we have introduced the trace of the DT:

$\operatorname{Tr}(\mathrm{D})=\sum_{\mathrm{k}=1}^{3} \lambda_{\mathrm{k}}=\sum_{\mathrm{k}=1}^{3} \mathrm{D}_{\mathrm{kk}}$

Fitting the expression ${ }^{[1]}$ while measuring a sufficient set of experimental points would allow the determination of the diffusion tensor elements $D_{\mathrm{ij}}$ and of the eigenvalues $\lambda_{\mathrm{i}}$, $(\mathrm{i}=1,2,3)$. The sorted eigenvalues (usually sorted in decreasing order $\lambda_{\mathrm{Si}}: \lambda_{\mathrm{S} 1}>\lambda_{\mathrm{S} 2}>\lambda_{\mathrm{S} 3}$ ) of the diffusion tensor are also known as the principal diffusivities.

\section{Eigenvectors}

Calculated sorted eigenvalues (diffusivities) $\lambda_{\mathrm{Si}(i=1,2,3)}$ : $\lambda_{\mathrm{S} 1}>\lambda_{\mathrm{S} 2}>\lambda_{\mathrm{S} 3}$ are rotationally invariant quantities; it is possible to define more intrinsic diffusion quantities for expressing the isotropic/anisotropic behavior of the 
diffusion phenomena within the brain tissue. Indeed the eigenvectors $\mathrm{v}_{1}, \mathrm{v}_{2}$, and $\mathrm{v}_{3}$ corresponding to each of the sorted eigenvalues $\lambda_{\mathrm{S} 1}>\lambda_{\mathrm{S} 2}>\lambda_{\mathrm{S} 3}$, respectively, are calculated according to the matrix algebra theory. In this comparative study, we assume that the eigenvector of the highest diffusivity $(\lambda \mathrm{S} 1)$ corresponds to the orientation of the fiber tracts the most probable within a pixel.

\section{Data acquisition of the diffusion-weighted magnetic resonance imaging}

The diffusion-weighted magnetic resonance images were obtained using a 1.5 Tesla whole body imager system (Magnetom Vision, Siemens, Erlangen, Germany). The system was supplied with an actively shielded gradient system. The maximum gradient strength was $25 \mathrm{mT} / \mathrm{m}$. A database of 8 normal volunteer's brains with age ranging from 25 to 34 years was all acquired with this protocol. All individuals consented to undergo the DTI study that was approved by the Local Ethics Committee.

All measurements were done using a manufacturer supplied head coil used for routine head clinical examinations. The DWI was performed using a single-shot DW echoplanar imaging sequence (DW-EPI) following a Stejskal-Tanner methodology. ${ }^{[17-18]}$

The MRI parameters were echotime: $\mathrm{TE}=123 \mathrm{~ms}$, field of view $=240 \mathrm{~mm}$, square matrix size $=128 \times 128$, slice thickness of $5 \mathrm{~mm}$. Each diffusion gradient lasted a duration $\delta=26 \mathrm{~ms}$, while the time between the start of each of both symmetrical consecutive diffusion gradients $\Delta=59.7 \mathrm{~ms}$. Rise times of the diffusion gradients were fixed to $\varepsilon=700 \mu$ s. The trapezoidal diffusion gradient strengths were 0,11 , and $22 \mathrm{mT} / \mathrm{m}$. The transverse slice orientation was chosen, which was perpendicular to the external magnetic field. . $^{[1,2,12,13,20,21]}$

The basic measurement duration lasted $38 \mathrm{~s}$, generating a series of 13 images. Each series included of a single image free of diffusion weighting, and one weakly and one strongly diffusion-weighted image for each of six noncollinear magnetic field gradient directions of frame of reference: $\{(0,0,0),(1,0,0),(0,1,0),(0,0,1),(1 / 21 / 2$, $1 / 21 / 2,0),(1 / 21 / 2,0,1 / 21 / 2),(0,1 / 21 / 2,1 / 21 / 2)\}$. The value of b-matrix components varied from 0.60 to $1225.05 \mathrm{~s} / \mathrm{mm}^{2} \cdot{ }^{[1,2,12,13,20,21]}$

In all experiments, the signal to noise ratio (SNR) of the DW-EPI images was increased by averaging over multiple acquisitions, which is commonly done in clinical practice. This was achieved by achieving the complete imaging experiment Nacq times (where Nacq is the number of acquisitions), then the average of the signal of these Nacq measurements was considered for the tensor calculations. As it is difficult to attach a single meaningful SNR to the 13 images comprising a diffusion tensor measurement, we preferred to report the results as a function of the Nacq, which is the same for all these images.

\section{Data postprocessing}

The echo-planar diffusion tensor image data were moved from the scanner to a personal computer running Linux for off-line postprocessing. We used C-programs that were developed in our group for this purpose..$^{[1,2,12,13,20,21]}$ From the 13 DWIs measured for the single slice considered, we derived the six independent components of the diffusion tensor. $^{[1,2,12,13,20,21]}$ After diagonalization, a sorting routine produced the sorted eigenvalues.

Finally, we calculated the three eigenvectors components corresponding to the sorted diffusivities. All calculations were done on the basis of pixel by pixel.

The first series of color map was based on the DWIs that are calculated using three principal colors, red, green, and blue; these colors are allocated in gray scale of the three DWIs, that corresponding to three orthogonal directions of the laboratory measurement frame of reference $\mathrm{x}, \mathrm{y}$, and $\mathrm{z}$, respectively.

The principal color images were then integrated pixel by pixel to form a unique color image.

The second series of color map was originated by allocating the three principal colors, red, blue, and green, respectively, to the images corresponding to three eigenvalues $\lambda_{1}$, $\lambda_{2}$, and $\lambda_{3}$, respectively, of the diffusion tensor. The apportionment of colors was such that the colors red, green, and blue corresponded to the first, the second, and the third eigenvalue calculated without sorting.

The third series of color maps was originated by allocating the three principal colors to the eigenvectors $v 1, v 2$, and $v 3$ corresponding to the sorted eigenvalues of the diffusion tensor red, blue, and green, respectively. The allocation was done that the colors red, green, and blue corresponded to the eigenvectors in $\mathrm{x}, \mathrm{y}$, and $\mathrm{z}$, respectively.

In all described color mapping methods, $\mathrm{x}, \mathrm{y}$, and $\mathrm{z}$ are the axes of the measuring DW image data that are also the laboratory frame of reference.

The obtained result is that each pixel has a unique color, related to diffusion anisotropy reflecting fiber orientation of the white matter. ${ }^{[1,2,12,13,20,21,26]}$

\section{Results and Discussion}

\section{White matter in diffusion tensor maps}

The color maps are offering significant information and evidences about the anatomical structures found in the white matter of the brain. Indeed all types of maps reproduced the available diffusion pathways that were outlined along the highly organized commissural fiber bundles in the splenium of corpus callosum [Figure 1A and B]. These tracts are connecting the posterior aspects of both cerebral hemispheres; these findings are consistent with known 

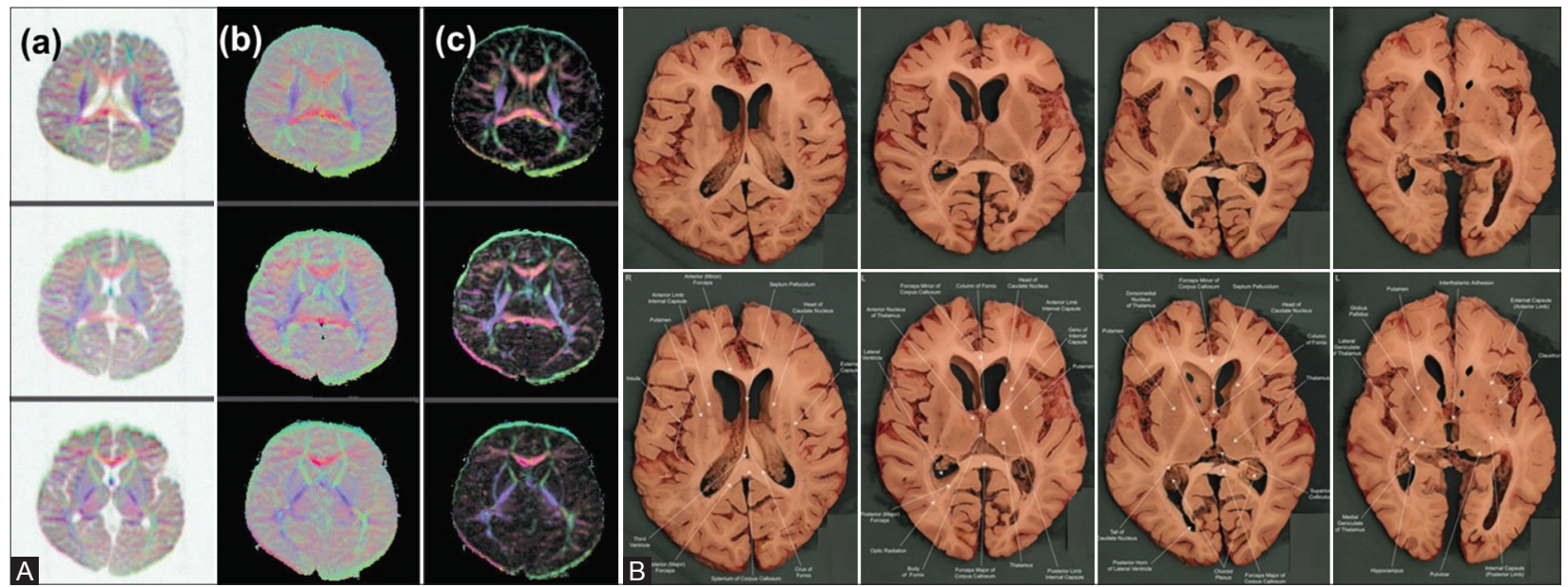

Figure 1: (A) Three slices of color maps of diffusion tracking fibers of the white matter. The columns represent: (a) Color maps are based on the diffusion-weighted magnetic resonance imaging that was achieved using three principal colors (red, green, and blue) allocated in gray scale of the three different diffusion-weighted images, corresponding to three orthogonal directions $\mathbf{x}, \mathbf{y}$, and $\mathbf{z}$, respectively, of the laboratory frame of reference. (b) Color maps are obtained by assigning the three principal colors (red, blue, and green), respectively, to the images corresponding to three derived eigenvalues $\lambda_{1}, \lambda_{2}$, and $\lambda_{3}$, respectively, of the diffusion tensor. (c) Color map obtained by assigning the three principal colors to eigenvectors $v_{1}, v_{2}$, and $v_{3}$ of sorted eigenvalues of the diffusion tensor. The color maps show the orientation of diffusion fiber tracks colored red and blue in the splenium of the corpus callosum. The colors reflected tracks that are best viewed in ( $c$ and b) respectively. The complete anatomical localization of tracks is displayed in the slice that crosses through the splenium. Tracks that traveled through the splenium were observed to split into two sets laterally and were color coded based on the pathway into the lateral. (B) Anatomical slices reflecting organizes brain tissue fibers are found in the color maps derived from diffusion tensor parameters and diffusion tensor magnetic resonance imaging derived parameters. The anatomical maps demonstrated the superiority of the eigenvectors maps in Figure 1A (c) since it demonstrates the perfect delimitation of the caudate nucleus, corpus callosum, splenium, and early optical nerves

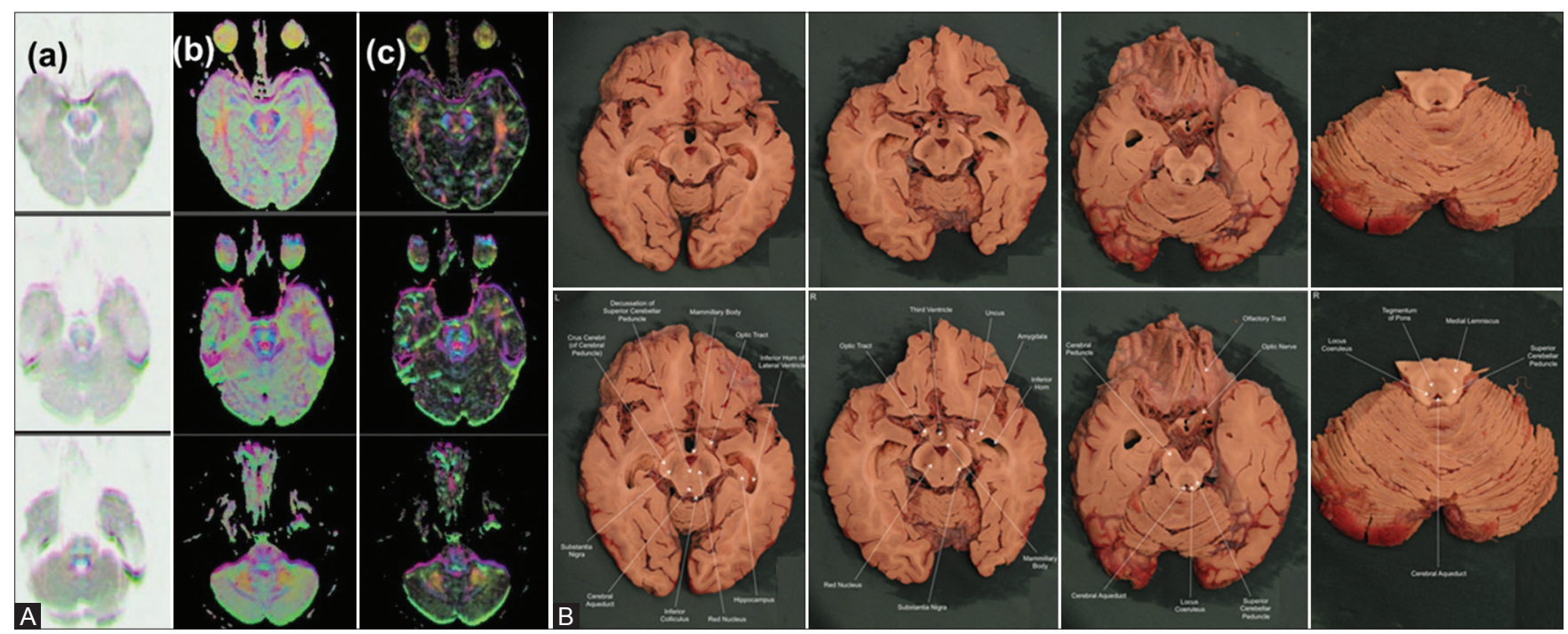

Figure 2: (A) Color maps are shown at the compartment level of the brain stem including (a) Color map based on the diffusion-weighted magnetic resonance imaging that was obtained using three principal colors (red, green, and blue) allocated in gray scale of the three diffusion-weighted images, corresponding to three orthogonal directions of the laboratory frame of reference $\mathbf{x}, \mathbf{y}$, and $\mathbf{z}$, respectively; (b) Color map derived by assigning the three principal colors (red, blue, and green), respectively, to the images corresponding to the eigenvalues $\lambda_{1}, \lambda_{2}$, and $\lambda_{3}$, respectively, of the Diffusion Tensor; and (c) Color map obtained by assigning the three primary colors to the eigenvectors $v_{1}, v_{2}$, and $v_{3}$ corresponding to the sorted eigenvalues of the diffusion tensor. The mapping results show major tracts within three different consecutive slice levels including the medulla, pons and midbrain, brain stem. (B) Anatomical slices reflecting organized brain tissue fibers found in the color maps derived from diffusion tensor parameters and diffusion tensor magnetic resonance imaging-derived parameters. The anatomical maps demonstrated the superiority of the eigenvectors maps in Figure $2 \mathrm{~A}$ (c) since it demonstrates the perfect delimitation of the medulla, pons, midbrain, optical nerves, and brain stem tracts

anatomy [Figures 1B, 2B and in references]; ${ }^{[31,32]}$ such available brain white matter fiber tracks are paralleled to the curvature of the splenium and divided lateral way to progress across several slices into the occipital cerebral lobe (forceps major, red tracks) and parietal lobe of the brain (blue tracks).
Figures $1 \mathrm{~A}$ and $2 \mathrm{~A}$ show nine representative slices of color maps obtained using the three color mapping approaches. It is well remarkable that the DTI-based color maps (columns b and c) demarcated within the brain white matter a more complex substructures compared to DWI-derived color maps (column a). The signal intensity in the color 
maps elucidates the magnitude of the diffusion anisotropy and provides higher differentiation of contrast between the brain's white matter and gray matter; in addition, the colors, which specified orientation of fiber tracts, were used to differentiate various tracts within the brain's white matter. This was best seen in the color maps based on the three eigenvectors corresponding to the sorted eigenvalues.

Reconstruction of brain anatomical features on diffusion tensor color-based maps can be more considered in 3D-trajectory data that would provide reconstruction of brain's white matter fibers; this would be possible using the eigenvector maps. In the DWI, the best-structured white matter tracts (e.g., splenium and corpus callosum) reflect the same findings on the maps of the eigenvalues and eigenvectors; this could be attributed to the accidental orientation of these structures along the axis of the frame of reference of the laboratory.

A change in orientation of this structure would reflect completely different color distribution on the maps, which has to correspond to the new orientation situations.

\section{Brain stem in diffusion tensor maps}

Scoping the observation on the central area of the color maps confirmed the same findings underlined the brain's white matter (previous section).

Figure $2 \mathrm{~A}$ is showing the results of tracks of the major brainstem fibers including corticospinal tract, medial lemniscus, middle cerebellar peduncle, and superior cerebellar peduncle. The color mapping result shows clearly the superiority of the eigenvector maps since this provided high qualitative conformity with known standard anatomical data [Figure 2B]. ${ }^{[31,32]}$

\section{Gray matter in diffusion tensor maps}

The gray matter is known for its isotropic diffusion characteristics; this was well reflected in the eigenvector maps. The DWI and the eigenvalue color maps which have areas (the cortical area) that are completely isotropic are still showing anisotropy features suggesting higher organization of this tissue [Figures 1 and 2].

When referring to the original information on which these color maps are based, three important remarks have to be recorded:

1. The color maps derived from DWI showed better colorful features which lead to more congenial images. Then again, these DW derived images are based on signal data that were contaminated by factors unconnected to diffusion such as T2-weighting and proton density; indeed the diffusion phenomena itself is not the best characterized by the DWIs along three spatial directions of axes that are used as starting data for all derived maps

2. The color maps based on the eigenvalues of the diffusion tensor offer information concerning the values of the diffusion in the diffusion frame of reference instead of the laboratory frame. Such is the case of derived maps from the three components of the diffusion tensor measured in the laboratory frame of reference

3. The color map derived from the eigenvectors of the sorted eigenvalues has expressed more explicit colors; it is established on a better characterization of the diffusion anisotropy and fiber tract orientation (full diffusion tensor). Therefore, it should be more reliable.

Diffusion tensor maps and general anatomical correlations

Indeed, the anatomical and DTI results showed high correlation of the white matter tracts showed in all DTI-derived maps. However, DT maps based on eigenvectors demonstrated best delineation of white matter fiber tracts followed by eigenvalues weighting maps and lastly by color maps-based diffusion-weighted MRI that was obtained using the three principal colors where red, green, and blue were allocated in gray scale of the three diffusion-weighted images, while corresponding to the three main orthogonal referential directions $\mathrm{x}, \mathrm{y}$, and $\mathrm{z}$, respectively.

Thus, the optimum anatomical delineation was obtained for the following white matter tissue where the maps did show the orientation of diffusion fiber tracks in red and blue within the splenium of the corpus callosum. The common anatomical localization of fiber tracks is shown in the slice that crosses through the splenium anatomical entity. Indeed, fiber tracks that traversed through the splenium were observed to split into two sets laterally and were color coded based on their pathway into the lateral side. Hence, the anatomical slices reflecting organized brain tissue fibers were found in the color maps derived from diffusion tensor parameters and diffusion tensor MRI-derived parameters. All anatomical maps demonstrated the superiority of the eigenvectors maps in Figure 1A since it demonstrates the perfect delimitation of the caudate nucleus, corpus callosum, splenium, and early optical nerves.

The mapping results showed major tracts at three consecutive slice levels such as the medulla, pons, and midbrain; this was well correlated to anatomical slices reflecting organized brain tissue fibers found in the color maps derived from diffusion tensor parameters and diffusion tensor MRI-derived parameters. The anatomical maps demonstrated the superiority of the eigenvectors maps in Figure 2A since it demonstrates the perfect delimitation of the medulla, pons, midbrain, optical nerves, and brain stem tracts [Figure 2B].

\section{Conclusions}

Indeed, an extreme existing correspondence is established between the DTI data in the color maps and the fiber tracts' orientation of known anatomical structures of brain tissue 
(e.g., corpus callosum and gray matter). In the meantime, rotationally invariant quantities such as the eigenvector components of the diffusion tensor reflected the best real orientation found in the studied tissue.

\section{Financial support and sponsorship}

None.

\section{Conflicts of interest}

There are no conflicts of interest.

\section{References}

1. Housni A, Boujraf S. Multimodal magnetic resonance imaging in the diagnosis and therapeutical follow-up of brain tumors. Neurosciences (Riyadh) 2013;18:3-10.

2. Boujraf S, Luypaert R, Shabana W, De Meirleir L, Sourbron S, Osteaux M, et al. Study of pediatric brain development using magnetic resonance imaging of anisotropic diffusion. Magn Reson Imaging 2002;20:327-36.

3. Meng $\mathrm{Y}$, Zhang $\mathrm{X}$. In vivo diffusion spectrum imaging of non-human primate brain: Initial experience in transcallosal fiber examination. Quant Imaging Med Surg 2014;4:129-35.

4. van Schouwenburg MR, Onnink AM, ter Huurne N, Kan CC, Zwiers MP, Hoogman M, et al. Cognitive flexibility depends on white matter microstructure of the basal ganglia. Neuropsychologia 2014;53:171-7.

5. Ellmore TM, Murphy SM, Cruz K, Castriotta RJ, Schiess MC. Averaging of diffusion tensor imaging direction-encoded color maps for localizing substantia nigra. Comput Biol Med 2014;51:104-10.

6. Mandelli ML, Berger MS, Bucci M, Berman JI, Amirbekian B, Henry RG, et al. Quantifying accuracy and precision of diffusion MR tractography of the corticospinal tract in brain tumors. J Neurosurg 2014;121:349-58.

7. Rachakonda T, Shimony JS, Coalson RS, Lieu JE. Diffusion tensor imaging in children with unilateral hearing loss: A pilot study. Front Syst Neurosci 2014;8:87.

8. Mills KL, Tamnes CK. Methods and considerations for longitudinal structural brain imaging analysis across development. Dev Cogn Neurosci 2014;9:172-90.

9. Lee VK, Nau AC, Laymon C, Chan KC, Rosario BL, Fisher C, et al. Successful tactile based visual sensory substitution use functions independently of visual pathway integrity. Front Hum Neurosci 2014;8:291.

10. Cherubini A, Péran P, Spoletini I, Di Paola M, Di Iulio F, Hagberg GE, et al. Combined volumetry and DTI in subcortical structures of mild cognitive impairment and Alzheimer's disease patients. J Alzheimers Dis 2010;19:1273-82.

11. Leow $\mathrm{AD}$, Zhu S, Zhan L, McMahon K, de Zubicaray GI, Meredith M, et al. The tensor distribution function. Magn Reson Med 2009;61:205-14.

12. Boujraf S, Luypaert R, Eisendrath H, Osteaux M. Echo planar magnetic resonance imaging of anisotropic diffusion in asparagus stems. Magn Reson Mater Phys Biol Med 2001;13:82-90.

13. Luypaert R, Boujraf S, Sourbron S, Osteaux M. Diffusion and perfusion MRI: Basic physics. Eur J Radiol 2001;38:19-27.
14. Park CH, Kou N, Boudrias MH, Playford ED, Ward NS. Assessing a standardised approach to measuring corticospinal integrity after stroke with DTI. Neuroimage Clin 2013;2:521-33.

15. Umesh Rudrapatna S, Wieloch $\mathrm{T}$, Beirup $\mathrm{K}$, Ruscher $\mathrm{K}$, Mol W, Yanev P, et al. Can diffusion kurtosis imaging improve the sensitivity and specificity of detecting microstructural alterations in brain tissue chronically after experimental stroke? Comparisons with diffusion tensor imaging and histology. Neuroimage 2014;97:363-73.

16. George E, Heier L, Kovanlikaya I, Greenfield J. Diffusion tensor imaging of pyramidal tract reorganization after pediatric stroke. Childs Nerv Syst 2014;30:1135-9.

17. Lai $\mathrm{C}$, Zhou $\mathrm{HC}$, Ma $\mathrm{XH}$, Zhang $\mathrm{H} \times$. Quantitative evaluation of the axonal degeneration of central motor neurons in chronic cerebral stroke with diffusion tensor imaging. Acta Radiol 2014;55:114-20.

18. Yamada K. Imaging of small brain infarctions. Brain Nerve 2013;65:783-8.

19. Yan X, Zhou M, Ying L, Yin D, Fan M, Yang G, et al. Evaluation of optimized b-value sampling schemas for diffusion kurtosis imaging with an application to stroke patient data. Comput Med Imaging Graph 2013;37:272-80.

20. Boujraf S, Luypaert R, Osteaux M. B matrix errors in echo planar diffusion tensor imaging. J Appl Clin Med Phys 2001;2:178-83.

21. Boujraf S. Strategies for assessing diffusion anisotropy on the basis of magnetic resonance images: Comparison of systematic errors. J Med Signals Sens 2014;4:85-93.

22. Pierpaoli C, Jezzard P, Basser PJ, Barnett A, Di Chiro G. Diffusion tensor MR imaging of the human brain. Radiology 1996;201:637-48.

23. Coremans J, Luypaert R, Verhelle F, Stadnik T, Osteaux M. A method for myelin fiber orientation mapping using diffusion-weighted MR images. Magn Reson Imaging 1994;12:443-54.

24. Basser PJ, Pierpaoli C. Microstructural and physiological features of tissues elucidated by quantitative-diffusion-tensor MRI. J Magn Reson B 1996;111:209-19.

25. Pierpaoli C, Basser PJ. Toward a quantitative assessment of diffusion anisotropy. Magn Reson Med 1996;36:893-906.

26. Virta A, Barnett A, Pierpaoli C. Visualizing and characterizing white matter fiber structure and architecture in the human pyramidal tract using diffusion tensor MRI. Magn Reson Imaging 1999; 17:1121-33.

27. Le Bihan D. Molecular diffusion nuclear magnetic resonance imaging. Magn Reson Q 1991;7:1-30.

28. Basser PJ, Mattiello J, LeBihan D. Estimation of the effective self-diffusion tensor from the NMR spin echo. J Magn Reson B 1994;103:247-54.

29. Mattiello J, Basser PJ, Le Bihan D. The $b$ matrix in diffusion tensor echo-planar imaging. Magn Reson Med 1997;37:292-300.

30. Basser PJ, Mattiello J, LeBihan D. MR diffusion tensor spectroscopy and imaging. Biophys J 1994;66:259-67.

31. Nieuwenhuys A, Voogd J, van Huijzen C. The Human Central Nervous System: A Synopsis and Atlas. Berlin: Springer; 1988.

32. Baert AL, Sator K. Imaging of Orbital and Visual Pathway Pathology. Berlin: Springer-Verlag; 2002. 


\section{BIOGRAPHIE}

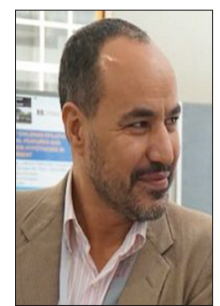

Saïd Boujraf MedSc, $\mathrm{PhD}$. is a Professor of Biophysics and Clinical MRI Methods, Department of Biophysics and Clinical MRI Methods, Faculty of Medicine and Pharmacy, University of Fez, Morocco and Director of the Clinical Neuroscience Laboratory, Faculty of Medicine and Pharmacy, University of Fez, Morocco.

Saïd Boujraf is a Medical Researcher graduated from 'Free University of Brussels' (VUB), Belgium.

Email: sboujraf@gmail.com 